\title{
Integrated analysis of differentially expressed profiles and construction of a competing endogenous long non-coding RNA network in renal cell carcinoma
}

\author{
Qianwei Xing ${ }^{1}$, Yeqing Huang ${ }^{1}$, You Wu ${ }^{1}$, Limin Ma ${ }^{1}$, Bo Cai ${ }^{\text {Corresp. }}{ }^{1}$ \\ ${ }^{1}$ Department of Urology, Affiliated Hospital of Nantong University, Nantong, Jiangsu, China \\ Corresponding Author: Bo Cai \\ Email address: caibo@ntu.edu.cn
}

Background: Long non-coding RNAs (IncRNAs) play crucial roles in the initiation and progression of renal cell carcinoma (RCC) by competing in binding to miRNAs, and related competitive endogenous RNA (ceRNA) networks have been constructed in several cancers. However, the coexpression network has been poorly explored in RCC. Methods: We collected RCC RNA expression profile data and relevant clinical features from The Cancer Genome Atlas (TCGA). A cluster analysis was explored to show different IncRNA expression patterns. Gene ontology (GO), Kyoto Encyclopedia of Genes and Genomes (KEGG) pathway enrichment analyses and gene set enrichment analysis (GSEA) were performed to analyze the functions of the intersecting mRNAs. Targetscan and miRanda bioinformatics algorithms were used to predict potential relationships among RNAs. Univariate Cox proportional hazards regression was conducted to determine the RNA expression levels and survival times. Results: Bioinformatics analysis revealed that the expression profiles of hundreds of aberrantly expressed IncRNAs, miRNAs, and mRNAs were significantly changed between different stages of tumors and non-tumor groups. By combining the data predicted by databases with intersection RNAs, a ceRNA network consisting of 106 IncRNAs, 26 miRNAs and 69 mRNAs was established. Additionally, a protein interaction network revealed the main hub nodes (VEGFA, NTRK2, DLG2, E2F2, MYB and RUNX1). Furthermore, 63 IncRNAs, 4 miRNAs and 31 mRNAs were significantly associated with overall survival. Conclusion: Our results identified cancer-specific IncRNAs and constructed a ceRNA network for RCC. A survival analysis related to the RNAs revealed candidate biomarkers for further study in RCC. 
1 Integrated analysis of differentially expressed profiles and construction of a

2 competing endogenous long non-coding RNA network in renal cell carcinoma

3 Qianwei Xing ${ }^{1}$, Yeqing Huang ${ }^{1}$, You Wu ${ }^{1}$, Limin Ma ${ }^{1}$, Bo Cai ${ }^{1}$

4

5

$6{ }^{1}$ Department of Urology, Affiliated Hospital of Nantong University, Nantong, 226001, China.

8 Qianwei Xing and Yeqing Huang contributed equally to this work.

9 Correspondence to: Bo Cai, email: caibo@ntu.edu.cn

Department of Urology, Affiliated Hospital of Nantong University, No.20 Xisi Road, Nantong, 226001, China.

11 E-mail: caibo@ntu.edu.cn

TEL: +08613814601551 
24 Abstract

25

26

27

Background: Long non-coding RNAs (lncRNAs) play crucial roles in the initiation and progression of renal cell carcinoma (RCC) by competing in binding to miRNAs, and related competitive endogenous RNA (ceRNA) networks have been constructed in several cancers. However, the coexpression network has been poorly explored in RCC.

Methods: We collected RCC RNA expression profile data and relevant clinical features from The Cancer Genome Atlas (TCGA). A cluster analysis was explored to show different lncRNA expression patterns. Gene ontology (GO), Kyoto Encyclopedia of Genes and Genomes (KEGG) pathway enrichment analyses and gene set enrichment analysis (GSEA) were performed to analyze the functions of the intersecting mRNAs. Targetscan and miRanda bioinformatics algorithms were used to predict potential relationships among RNAs. Univariate Cox proportional hazards regression was conducted to determine the RNA expression levels and survival times.

Results: Bioinformatics analysis revealed that the expression profiles of hundreds of aberrantly expressed lncRNAs, miRNAs, and mRNAs were significantly changed between different stages of tumors and non-tumor groups. By combining the data predicted by databases with intersection RNAs, a ceRNA network consisting of 106 lncRNAs, 26 miRNAs and 69 mRNAs was established. Additionally, a protein interaction network revealed the main hub nodes (VEGFA, NTRK2, DLG2, E2F2, MYB and RUNX1). Furthermore, 63 lncRNAs, 4 miRNAs and 31 mRNAs were significantly associated with overall survival.

Conclusion: Our results identified cancer-specific lncRNAs and constructed a ceRNA network for RCC. A survival analysis related to the RNAs revealed candidate biomarkers for further study in RCC. 
51

52

53

54

55

56

57

58

59

60

61

62

\section{Introduction}

Renal cell carcinoma (RCC) is among the ten leading cancer types for estimated new cancer cases in both males and females in the United States (Siegel, Miller \& Jemal, 2017). Although the 5-year relative survival rate for all cancers has increased due to advances in diagnosis and therapy over the past 3 decades, discriminative biological markers have not been determined in RCC for early-stage diagnosis and therapy, unlike for other cancers. Increasing numbers of studies on RCC have been conducted to explore tumor initiation and progression, including protein-coding RNAs (mRNAs) and non-coding RNAs (ncRNAs) (Frew \& Moch, 2015; Lorenzen \& Thum, 2016; Martens-Uzunova et al., 2014; Qin et al., 2014; Schmidt \& Linehan, 2016); however, the tumor-specific mechanisms in the regulation of tumor progression and biological behaviors are not fully understood. Recently, long non-coding RNAs (lncRNAs) have been reported to play important roles in the molecular mechanism of RCC (Chen et al., 2016; Li, Shuch \& Gerstein, 2017; Serghiou, Kyriakopoulou \& Ioannidis, 2016).

LncRNAs are a subtype of ncRNAs with transcript lengths over 200 nucleotides and were once viewed as transcriptional noise without the capacity to encode proteins (Guttman et al., 2009). However, growing evidence suggests that lncRNAs may play crucial biological roles in transcriptional regulation, cellular development, and RNA modification (Schmitt \& Chang, 2016). Emerging reports have revealed that lncRNAs are associated with various human diseases, such as neurological diseases (Bai et al., 2014; Lau, Frigerio \& De Strooper B, 2014), cardiovascular diseases (Vausort, Wagner \& Devaux, 2014; Yang et al., 2014), immune inflammatory diseases (Kino et al., 2010), and malignant tumors; moreover, lncRNAs can be drug targets (Sana et al., 2012). In urologic malignancies, IncRNAs are thought to be related to tumor formation, invasion, and metastasis (Martens-Uzunova et al., 2014). In RCC, several studies have reported that lncRNAs may function as oncogenes or tumor suppressors and that they can affect the long-term survival and mortality of patients. For example, TRIM52-AS1 overexpression can suppress cell migration and proliferation and can induce apoptosis of RCC (Liu et al., 2016). Additionally, down-regulated lncRNA H19 inhibits RCC carcinogenesis (Wang et al., 2015). Tumor formation and development are complex pathophysiological processes, and lncRNAs may interact with miRNAs or mRNAs or other molecules in regulatory capacities. There are multiple hypotheses regarding how cancer-specific molecules communicate with each other (Gomes, Nolasco \& Soares, 2013; Huang et al., 2017).

In 2011, Salmena et al. proposed a competing endogenous RNA (ceRNA) hypothesis 
83

84

101

102

103

(Salmena et al., 2011), which indicated that all types of RNA transcripts communicate with each other by competing for binding to miRNA response elements (MREs). This competition between mRNAs, lncRNAs, pseudogenes, and circular RNAs widely exists in tumor initiation and progression. Xiao $\mathrm{H}$ et al.reported that the lncRNA MALATI participates in the ceRNA network by sponging miR-200s to regulate ZEB2 expression (Xiao et al., 2015). Additionally, Qu L et al. demonstrated that exosome-transmitted lncARSR acts as a ceRNA to promote sunitinib resistance in renal cancer ( $Q u$ et al., 2016). Nevertheless, studies incorporating large sample sizesand high-throughput detection methods to identify whether lncRNAs are correlated with overall survival, gender, or other clinical features or to determine the ceRNA mechanisms of lncRNAs with aberrant expression in RCC are currently lacking.

The Caner Genome Atlas (TCGA) (http://cancergenome.nih.gov) is a platform that can provide clinical information and RNA sequencing with miRNA, IncRNA, and mRNA data. We downloaded data sets from the TCGA that included RNA sequences of different stage (according to the American joint committee on cancer, AJCC) tumor tissues and adjacent non-tumor renal tissues. To the best of our knowledge, this study is the first to use this large sequencing database to investigate the differential expression profiles of a cancer-specific lncRNA and ceRNA coexpression network in RCC. This new approach of predicting caner-specific lncRNAs and their ceRNA potential can help us elucidate the functions of lncRNAs in RCC.

\section{Materials and methods}

\section{Patients and samples}

A total of 537 patients with RCC were retrieved from the TCGA data portal. We selected data according to the following criteria: 1) the histopathological diagnosis was RCC; 2) complete data was required for all samples of all stages; and 3) the included patients had no other malignancies. Overall, a total of 457 RCC patients were enrolled in this study. Among these samples, we analyzed miRNA sequences from 64 adjacent non-tumor tissue samples and 213 tumor tissue samples (95 stage I samples, 27 stage II samples, 46 stage III samples, and 45 stage IV samples)and mRNA/lncRNA sequences from 65 adjacent non-tumor tissues and 454 tumor tissues (209 stage I samples, 51 stage II samples, 114 stage III samples, and 80 stage IV samples). This study was conducted in accordance with the publication guidelines provided by the TCGA (http://cancergenome.nih.gov/publications/publicationguidelines); therefore, further approval of the ethics committee was not required. 
115

116

117

118

119

120

121

122

123

124

125

126

127

128

129

130

131

132

133

134

135

136

137

138

139

140

141

142

143

144

145

146

\section{RNA sequencing data sets and computational analysis}

The RCC RNA expression profile data (level 3) and relevant clinical features were acquired from the TCGA data portal (through Feb, 2017). The lncRNA and mRNA expression profiles were normalized using the RNASeqV2 system provided by the TCGA database. The miRNA expression profiles, downloaded from the TCGA database, were obtained using the Illumina HiSeq 2000 miRNA sequencing platform (Illumina Inc., USA). No further normalization was needed, as the TCGA database already had normalized the lncRNA, miRNA, and mRNA expression profile data. Then, we compared differentially expressed RNAs at 4 levels, including stage I RCC patient tumor tissues vs. non-tumor renal tissues, AJCC stage II RCC patient tumor tissues vs. non-tumor renal tissues, AJCC stage III RCC patient tumor tissues vs. non-tumor renal tissues, and AJCC stage IV RCC patient tumor tissues vs. non-tumor renal tissues. In the next step, we collected intersecting lncRNAs $(\mathrm{P} \leq 0.01, \mathrm{FDR} \leq 0.01, \mathrm{FC} \geq 2)$, miRNAs $(\mathrm{P}<0.05$, $\mathrm{FDR}<0.05, \mathrm{FC} \geq 2)$ and mRNAs ( $\mathrm{P} \leq 0.01, \mathrm{FDR} \leq 0.01, \mathrm{FC} \geq 3$ ) for hierarchical clustering and further bioinformatics analysis. A flow chart was depicted to show the framework of this study (Figure 1).

\section{GO, KEGG pathway analysis and GSEA}

Gene ontology (GO) analysis contains three domains: biological processes, cellular components, and molecular functions. We used a GO database to analyze differentially expressed intersecting mRNAs (http://www.geneontology.org). The molecular functions of upand down-regulated genes were identified. The potential functions of the aberrantly expressed genes involved in the pathways were analyzed using the Kyoto Encyclopedia of Genes and Genomes (KEGG) (http://www.kegg.jp). Besides, The above data were analyzed using gene set enrichment analysis (GSEA) (Broad Institute; MA, USA, accessed at http://software.broadinstitute.org/gsea/). Additionally, we identified intersecting mRNAs $(\mathrm{P}<0.05, \mathrm{FDR}<0.05)$ by combing GO with KEGG analyses to build a ceRNA network.

\section{Construction of a IncRNA-related ceRNA network}

According to the ceRNA hypothesis, IncRNA can regulate mRNA expression by sequestering and binding miRNAs like miRNA sponges. We constructed the ceRNA network through three steps: 1) we identified intersecting lncRNAs, miRNAs, and mRNAs that were differentially expressed in four stages (including up- and down-regulation); 2) we predicted IncRNA-miRNA interactions by miRanda (http://www.microrna.org/microrna/home.do) and used the Targetscan (http://www.targetscan.org) and miRanda databases to find target genes; and 3) we 
147 integrated aberrantly expressed data from the TCGA and the predicted miRNA information.

148 Through these steps, we constructed a ceRNA network with intersecting lncRNAs and mRNAs 149 that were negatively regulated by miRNAs. Additionally, a figure for the ceRNA network was 150 depicted using Cytoscape v3.0.

151 PPI network analysis

152 To clarify the potential relationships between the aberrantly expressed genes and RCC, we 153 performed a protein-protein interaction (PPI) network analysis with the online software String 154 (score $>0.4)$.

\section{Statistical analysis}

\section{Results}

\section{Cancer-specific IncRNAs in RCC}

To compare stage I RCC tumor tissues and adjacent non-tumor RCC tissues from the TCGA database, we found 354 differentially expressed lncRNAs $(P \leq 0.01, F D R \leq 0.01, F C \geq 2)$. Similarly, we identified 357 differentially expressed lncRNAs between stage II RCC tumor tissues and adjacent non-tumor RCC tissues. Three hundred eighty-five differentially expressed lncRNAs were identified between stage III RCC tumor tissues and non-tumor RCC tissues, and 404 differentially expressed lncRNAs were identified between stage IV RCC tumor tissues and non-tumor RCC tissues. Then, we found 291 intersecting lncRNAs from the above lncRNAs (92 up-regulated; 199 down-regulated) (Figure 2A, Supplementary Table 1). A heat map of these 291 aberrant lncRNAs was established using R software (Supplementary Figure 1), which revealed different expression patterns of lncRNAs in RCC.

\section{GO, KEGG pathway analyses and GSEA}

A total of 2,650 mRNAs $(P \leq 0.01, F D R \leq 0.01, F C \geq 3)$ were differentially expressed between stage I RCC tumors and non-tumor RCC tissues from the TCGA database, 2,683 mRNAs were differentially expressed between stage II RCC tumors and non-tumor RCC tissues, 2,987 
179

180

181

182

183

184

185

186

187

188

189

190

191

192

193

194

195

196

197

198

199

200

201

202

203

204

205

206

207

208

209

210

mRNAs were differentially expressed between stage III RCC tumors and non-tumor RCC tissues, and 3,031 mRNAs were differentially expressed between stage IV RCC tumors and non-tumor RCC tissues. Next, we identified 2,269 intersecting mRNAs (Figure 2C) from the above mRNAs (1,132 up-regulated; 1,147 down-regulated).

We further explored the biological functions of these up- and down-regulated proteincoding RNAs by GO and KEGG pathway enrichment analyses. Using a GO database for comprehensive analysis, we found that the top three processes of the up-regulated mRNAs involved immune responses, signal transduction, and inflammatory responses, while the top three processes of the down-regulated mRNAs were transmembrane transport, small molecule metabolic processes, and excretion (Figure 3A). The significant pathways of the up-regulated mRNAs included cytokine-cytokine receptor interactions, Staphylococcus aureus infection, and cell adhesion molecules, which were determined by KEGG pathway enrichment analysis (Figure 3B).The pathways correlated with the down-regulated mRNAs included metabolic pathways, neuroactive ligand-receptor interactions, protein digestion, and absorption(Figure 3B). In addition, GSEA of up and down-regulated mRNAs was implemented using the gene sets of GO and KEGG pathway database (Supplementary Table 2, Supplementary Table 3, Supplementary Table 4, Supplementary Table 5). Finally, we found 738 significant intersecting mRNAs by GO and KEGG analyses $(\mathrm{P}<0.05$, FDR $<0.05)$.

\section{Construction of the ceRNA network}

We found 132 RCC-related miRNAs that were differentially expressed between stage I tumors and non-tumor tissues $(\mathrm{P}<0.05, \mathrm{FDR}<0.05, \mathrm{FC} \geq 2)$. Additionally, 144 miRNAs were differentially expressed between stage II tumors and non-tumor tissues, 138 miRNAs were differentially expressed between stage III tumors and non-tumor tissues, and 152 miRNAs were differentially expressed between stage IV tumor and non-tumor tissues. Subsequently, we identified 102 differentially expressed miRNAs from the above four groups (41 up-regulated; 61 down-regulated) (Figure 2B).

Integrating 291 intersecting IncRNAs and 102 intersecting miRNAs with those that we identified from the prolife TCGA data, we finally chose 106 lncRNAs that were negatively related to 26 miRNAs that were predicted by the miRanda database. These 26 miRNAs were negatively related to 69 mRNAs following a comparison of data predicted by Targetscan and miRanda with the data from GO and KEGG analyses. Based on these data, we established a miRNA-lncRNA-mRNA ceRNA network (Figure 4). 
211

212

213

214

215

216

217

218

219

220

221

222

223

224

225

226

227

228

229

230

231

232

233

234

235

236

237

238

239

240

241

242

\section{Construction of the PPI}

To better understandthe critical genes predicted in RCC, we established a PPI network that included 44 genes with scores of $>0.4$ in String (Figure 5). From the figure, we determined that the main hub nodes were VEGFA, NTRK2, DLG2, E2F2, MYB and RUNX1. Hence, the key genes associated with RCC can be predicted by our network.

\section{RNAs in the ceRNA network are related to survival}

To identify the miRNAs associated with prognosis, all miRNAs in the ceRNA network were analyzed via univariate Cox proportional hazards regression, and four miRNAs (miR-9-5p, miR-21-5p, miR-155-5p, and miR-244-5p) were observed to be significantly changed (log-rank $\mathrm{P}<0.05$ ). Additionally, we found 63 lncRNAs and $31 \mathrm{mRNAs}$ that were significantly related to overall survival (Supplementary Table 6). Here, we show figures for RNAs (Figure 6), as the coexpression profiles of RNAs inthe lncRNA-miRNA-mRNA correlations were all significantly associated with overall survival. These relationships are shown in Table 1.

\section{Discussion}

LncRNAs are emerging as having crucial roles in various cell biological processes and diverse malignant tumors (Martens-Uzunova et al., 2014; Schmitt \& Chang, 2016). Although their exact mechanisms are unclear, a variety of theories and hypotheses have been described regarding how lncRNAs participate in regulating malignant biological behavior. Some studies have documented that IncRNAs can affect cancer-related mRNAs by interacting with miRNAs, yielding new perspectives regarding lncRNA functions (Lv et al., 2016; Yue et al., 2016). This hypothesis has been supported in vivo and in vitro; moreover, ceRNA coexpression networks have been established for several human tumors (Li et al., 2016; Liu et al., 2016; Wang et al., 2016; Yang et al., 2016). A few studies have reported interactions between lncRNAs and miRNAs in RCC. Furthermore, one previous study suggested that some lncRNAs are associated with chromophobe RCC and that they may function as ceRNAs in complex ceRNA networks (He et al., 2016). Nevertheless, coexpression networks based on large-scale bioinformatics data and the potential roles of lncRNAs in ceRNA networks are still poorly understood in RCC. In the present study, we confirmed aberrantly expressed lncRNAs, miRNAs and mRNAs based on the RNA expression profiles of 457 RCC patient tissue samples from the TCGA. Additionally, we constructed a ceRNA network with cancer-specific lncRNAs, miRNAs and mRNAs. To understand the systematic biological roles of the abnormally expressed RNAs, we further 
243 investigated the association between these RNAs and overall survival, and we also constructed a 244 PPI network to find key mRNAs.

245 After analyzing the RNA sequencing data from the TCGA, we finally identified 291 cancer246 specific lncRNAs that were abnormally expressed in different stages of RCC. We then

247

248

249

250

251

252

253

254

255

256

257

258

259

260

261

262

263

264

265

266

267

268

269

270

271

272

273

274 constructed a cluster analysis map to show the differential distributions of the 291 lncRNAs in RCC tumor tissues and adjacent non-tumor tissues. Among these lncRNAs, several known lncRNAs, such as GAS5, CASC2, TCL6, MALAT-1, UCA1 and HOTAIR, have been found to be related to RCC cell proliferation, invasion and apoptosis, and they are important regulators in RCC occurrence and progression (Cao et al., 2016; Chen et al., 2017; Hong et al., 2017; Li et al., 2016; Qiao et al., 2013; Su et al., 2017). Furthermore, the massive RNA sequencing data identified in the present study revealed hundreds of differentially expressed lncRNAs that have not yet been reported and may function as novel oncogenes and theranostic markers in RCC.

The GO and KEGG pathway analyses of the 2,269 the intersecting mRNAs revealed the main biological processes and pathways in RCC. Many of them were classical pathways comprising important areas of RCC research, such as immune responses, signal transduction, and metabolism (Labrousse-Arias et al., 2017; Sakai, Miyake \& Fujisawa, 2013; Wettersten et al., 2017). However, some of biological processes and pathways, such as protein digestion and absorption, were reported in other malignancies (Dong et al., 2017; Li et al., 2017). From our enrichment analysis of large-scale samples focusing on RCC, we discovered multiple processes involved in neoplasm formation, which was supplementary to the results of previous studies. To enhance the validity and reliability of the ceRNA network, we selected the mRNAs that significantly participatedin both the GO and KEGG pathways for further analyses.

To provide a comprehensive view on how these lncRNAs participate in multiple biological processes, we constructed a ceRNA network combining the intersecting miRNAs and mRNAs, as many studies have elucidated the possibility that lncRNAs can be ceRNA regulators andcan communicate with other RNA transcripts (Lv et al., 2016; Qu et al., 2016; Salmena et al., 2011; Xiao et al., 2015; Yue et al., 2016). To increase the accuracy of the ceRNA network, we integrated the intersecting RNAs in different stages of RCC and evaluated the relationships between lncRNAs and miRNAs and between miRNAs and mRNAsthat were predicted by the Targetscan and miRanda databases. Ultimately, 106 lncRNAs, 26 miRNAs and 69 mRNAs were involved in the ceRNA network. Recent studies have demonstrated that down-regulation of the lncRNA CASC2 by miRNA-21 increases RCC cell proliferation and migration (Cao et al., 2016) 
275

276

277

278

279

280

281

282

283

284

285

286

287

288

289

290

291

292

293

294

295

296

297

298

299

300

301

302

303

304

305

and that MALT1 promotes aggressive RCC through Ezh2 and interacts with miR-205 (Hirata et al., 2015). The key IncRNAs identified above were also found in our ceRNA network. Additionally, our study integratively predicted the potential relationships between novel lncRNAs, miRNAs and mRNAs in RCC. Construction of a PPI network increases the reliability of our ceRNA network in the following ways: First, some genes described as main hub nodes, such as VEGFA, NTRK2, DLG2, E2F2, MYB and RUNX1, are themselves vital genes in human tumors, and some of them are directly associated with RCC (Chimge et al., 2016; Gao et al., 2016; Gonda \& Ramsay, 2016; Jones et al., 2013; Ma et al., 2015; Zubakov, Stupar \& Kovacs, 2006).Second, some genes in the ceRNA network that have not been reported to be correlated with RCC may also be oncogenes, as they can interact with RCC-related genes. The reasons above validate the hypotheses that the genes in the ceRNA network caninfluence RCC and that lncRNAs can be regulators that modulate oncogene expression.

To further evaluate the roles of RNAs in RCC diagnosis and prognosis, we analyzed the associations between RNAs in the ceRNA network and patient survival. We found 4 miRNAs, 63 lncRNAs and 31 mRNAs that were significantly correlated with overall RCC survival. Upregulation of the lncRNA MALAT1 has been reported to correlate with tumor progression and poor prognosis in RCC (Zhang et al., 2015), while some lncRNAs have been reported in other tumors, such as HOXA11-AS, which was regarded as a biomarker of poor progression in glioma (Wang et al., 2016). However, most of the 19 lncRNAs have not yet been reported. Subsequently, we found some RNAs in the lncRNA-miRNA-mRNA correlations that were uniformly related to overall survival, such as miR-21-5p-regulated mRNA KCNK10 and lncRNA TRHDE-AS1, which confirmed the internal relationships constructed in the ceRNA network in the present study.

Our work identified many valuable RNAs that are differentially expressed in RCC tissues via bioinformatics analysis. Some of these RNAs have been verified in vivo and in vitro experiments. However, most of the aberrant RNAs still need to be validated, and our ceRNA network, which was constructed in silico, needs to be validated with additional biological experiments.

\section{Conclusion}

The present study successfully identified hundreds of differentially expressed lncRNAs, 
306

307

308

309

310

311

312

313

314

315

317

318

319

320

321

322

323

324

325

326

327

328

329

330

331

332

333

334

335

336

337

338

339

340

miRNAs and mRNAs in RCC by bioinformatics analysis from candidate data from the TCGA. Moreover, we determined the biological processes and pathways via GO and KEGG pathway analyses with cancer-specific mRNAs in RCC. Importantly, we constructed a ceRNA network to explore the potential roles of lncRNAs in RCC, which can serve as a reference for further research. We also investigated the associations between RNAs and overall survival and found that some of the RNAs could be used as biomarkers for RCC diagnosis and prognosis.

\section{Acknowledgments}

We thank The Cancer Genome Atlas (TCGA) project and its contributors for this valuable public data set

\section{REFERENCES}

Bai Y, Nie S, Jiang G, Zhou Y, Zhou M, Zhao Y, Li S, Wang F, Lv Q, Huang Y, Yang Q, Li Q, Li Y, Xia Y, Liu Y, Liu J, Qian J, Li B, Wu G, Wu Y, Wang B, Cheng X, Yang Y, Ke T, Li H, Ren X, Ma X, Liao Y, Xu C, Tu X, Wang QK. 2014. Regulation of CARD8 expression by ANRIL and association of CARD8 single nucleotide polymorphism rs2043211 (p.C10X) with ischemic stroke. Stroke 45:383-388 DOI 10.1161/STROKEAHA.113.003393.

Cao Y, Xu R, Xu X, Zhou Y, Cui L, He X. 2016. Downregulation of lncRNA CASC2 by microRNA-21 increases the proliferation and migration of renal cell carcinoma cells. Molecular medicine reports 14:1019-1025 DOI $10.3892 / \mathrm{mmr} .2016 .5337$.

Chen J, Chen Y, Gu L, Li X, Gao Y, Lyu X, Chen L, Luo G, Wang L, Xie Y, Duan J, Peng C, Ma X. 2016. LncRNAs act as prognostic and diagnostic biomarkers in renal cell carcinoma: a systematic review and meta-analysis. Oncotarget 7:74325-74336 DOI 10.18632/oncotarget.11101.

Chen S, Ma P, Zhao Y, Li B, Jiang S, Xiong H, Wang Z, Wang H, Jin X, Liu C. 2017. Biological function and mechanism of MALAT-1 in renal cell carcinoma proliferation and apoptosis: role of the MALAT-1-Livin protein interaction. The journal of physiological sciences : JPS 67:577-585 DOI 10.1007/s12576-016-0486-8.

Chimge NO, Little GH, Baniwal SK, Adisetiyo H, Xie Y, Zhang T, O'Laughlin A, Liu ZY, Ulrich P, Martin A, MhawechFauceglia P, Ellis MJ, Tripathy D, Groshen S, Liang C, Li Z, Schones DE, Frenkel B. 2016. RUNX1 prevents oestrogen-mediated AXIN1 suppression and $\beta$-catenin activation in ER-positive breast cancer. Nature communications 7:10751 DOI 10.1038/ncomms10751.

Dong LF, Xu SY, Long JP, Wan F, Chen YD. 2017. RNA-Sequence Analysis Reveals Differentially Expressed Genes (DEGs) in Patients Exhibiting Different Risks of Tumor Metastasis. Medical science monitor : international medical journal of experimental and clinical research 23:2842-2849.

Frew IJ, Moch H. 2015. A clearer view of the molecular complexity of clear cell renal cell carcinoma. Annual review of pathology 10:263-289 DOI 10.1146/annurev-pathol-012414-040306.

Gao Y, Ma X, Yao Y, Li H, Fan Y, Zhang Y, Zhao C, Wang L, Ma M, Lei Z, Zhang X. 2016. miR-155 regulates the 
proliferation and invasion of clear cell renal cell carcinoma cells by targeting E2F2. Oncotarget 7:20324-20337 DOI 10.18632/oncotarget.7951.

Gomes AQ, Nolasco S, Soares H. 2013. Non-coding RNAs: multi-tasking molecules in the cell. International journal of molecular sciences 14:16010-16039 DOI 10.3390/ijms140816010.

Gonda TJ, Ramsay RG. 2016. Adenoid Cystic Carcinoma Can Be Driven by MYB or MYBL1 Rearrangements: New Insights into MYB and Tumor Biology. Cancer discovery 6:125-127 DOI 10.1158/2159-8290.CD-15-1470.

Guttman M, Amit I, Garber M, French C, Lin MF, Feldser D, Huarte M, Zuk O, Carey BW, Cassady JP, Cabili MN, Jaenisch R, Mikkelsen TS, Jacks T, Hacohen N, Bernstein BE, Kellis M, Regev A, Rinn JL, Lander ES. 2009. Chromatin signature reveals over a thousand highly conserved large non-coding RNAs in mammals. Nature 458:223-227 DOI 10.1038/nature07672.

He HT, Xu M, Kuang Y, Han XY, Wang MQ, Yang Q. 2016. Biomarker and competing endogenous RNA potential of tumorspecific long noncoding RNA in chromophobe renal cell carcinoma. OncoTargets and therapy 9:6399-6406 DOI 10.2147/OTT.S116392.

Hirata H, Hinoda Y, Shahryari V, Deng G, Nakajima K, Tabatabai ZL, Ishii N, Dahiya R. 2015. Long Noncoding RNA MALAT1 Promotes Aggressive Renal Cell Carcinoma through Ezh2 and Interacts with miR-205. Cancer research 75:1322-1331 DOI 10.1158/0008-5472.CAN-14-2931.

Hong Q, Li O, Zheng W, Xiao WZ, Zhang L, Wu D, Cai GY, He JC, Chen XM. 2017. LncRNA HOTAIR regulates HIF1 $\alpha$ /AXL signaling through inhibition of miR-217 in renal cell carcinoma. Cell death \& disease 8:e2772 DOI 10.1038/cddis.2017.181.

Huang X, Xiao R, Pan S, Yang X, Yuan W, Tu Z, Xu M, Zhu Y, Yin Q, Wu Y, Hu W, Shao L, Xiong J, Zhang Q. 2017. Uncovering the roles of long non-coding RNAs in cancer stem cells. Journal of hematology \& oncology 10:62 DOI 10.1186/s13045-017-0428-9.

Jones DT, Hutter B, Jäger N, Korshunov A, Kool M, Warnatz HJ, Zichner T, Lambert SR, Ryzhova M, Quang DA, Fontebasso AM, Stütz AM, Hutter S, Zuckermann M, Sturm D, Gronych J, Lasitschka B, Schmidt S, Seker-Cin H, Witt H, Sultan M, Ralser M, Northcott PA, Hovestadt V, Bender S, Pfaff E, Stark S, Faury D, Schwartzentruber J, Majewski J, Weber UD, Zapatka M, Raeder B, Schlesner M, Worth CL, Bartholomae CC, von KC, Imbusch CD, Radomski S, Lawerenz C, van Sluis P, Koster J, Volckmann R, Versteeg R, Lehrach H, Monoranu C, Winkler B, Unterberg A, Herold-Mende C, Milde T, Kulozik AE, Ebinger M, Schuhmann MU, Cho YJ, Pomeroy SL, von DA, Witt O, Taylor MD, Wolf S, Karajannis MA, Eberhart CG, Scheurlen W, Hasselblatt M, Ligon KL, Kieran MW, Korbel JO, Yaspo ML, Brors B, Felsberg J, Reifenberger G, Collins VP, Jabado N, Eils R, Lichter P, Pfister SM. 2013. Recurrent somatic alterations of FGFR1 and NTRK2 in pilocytic astrocytoma. Nature genetics 45:927-932 DOI 10.1038/ng.2682.

Kino T, Hurt DE, Ichijo T, Nader N, Chrousos GP. 2010. Noncoding RNA gas5 is a growth arrest- and starvation-associated repressor of the glucocorticoid receptor. Science signaling 3:ra8 DOI 10.1126/scisignal.2000568.

Labrousse-Arias D, Martínez-Alonso E, Corral-Escariz M, Bienes-Martínez R, Berridy J, Serrano-Oviedo L, Conde E, García-Bermejo ML, Giménez-Bachs JM, Salinas-Sánchez AS, Sánchez-Prieto R, Yao M, Lasa M, Calzada MJ. 2017. VHL promotes immune response against renal cell carcinoma via NF-kB-dependent regulation of VCAM-1. The Journal of cell biology 216:835-847 DOI 10.1083/jcb.201608024.

Lau P, Frigerio CS, De Strooper B. 2014. Variance in the identification of microRNAs deregulated in Alzheimer's disease and possible role of lincRNAs in the pathology: the need of larger datasets. Ageing research reviews 17:43-53 DOI 10.1016/j.arr.2014.02.006. 
Li CY, Liang GY, Yao WZ, Sui J, Shen X, Zhang YQ, Peng H, Hong WW, Ye YC, Zhang ZY, Zhang WH, Yin LH, Pu YP. 2016. Integrated analysis of long non-coding RNA competing interactions reveals the potential role in progression of human gastric cancer. International journal of oncology 48:1965-1976 DOI 10.3892/ijo.2016.3407.

Li S, Shuch BM, Gerstein MB. 2017. Whole-genome analysis of papillary kidney cancer finds significant noncoding alterations. PLoS genetics 13:e1006685 DOI 10.1371/journal.pgen.1006685.

Li X, Dong W, Qu X, Zhao H, Wang S, Hao Y, Li Q, Zhu J, Ye M, Xiao W. 2017. Molecular dysexpression in gastric cancer revealed by integrated analysis of transcriptome data. Oncology letters 13:3177-3185 DOI 10.3892/ol.2017.5798.

Li Y, Wang T, Li Y, Chen D, Yu Z, Jin L, Ni L, Yang S, Mao X, Gui Y, Lai Y. 2016. Identification of long-non coding RNA UCA1 as an oncogene in renal cell carcinoma. Molecular medicine reports 13:3326-3334 DOI 10.3892/mmr.2016.4894.

Liu D, Yu X, Wang S, Dai E, Jiang L, Wang J, Yang Q, Yang F, Zhou S, Jiang W. 2016. The gain and loss of long noncoding RNA associated-competing endogenous RNAs in prostate cancer. Oncotarget 7:57228-57238 DOI 10.18632/oncotarget.11128.

Liu Z, Yan HY, Xia SY, Zhang C, Xiu YC. 2016. Downregulation of long non-coding RNA TRIM52-AS1 functions as a tumor suppressor in renal cell carcinoma. Molecular medicine reports 13:3206-3212 DOI 10.3892/mmr.2016.4908.

Lorenzen JM, Thum T. 2016. Long noncoding RNAs in kidney and cardiovascular diseases. Nature reviews. Nephrology 12:360-373 DOI 10.1038/nrneph.2016.51.

Lv J, Fan HX, Zhao XP, Lv P, Fan JY, Zhang Y, Liu M, Tang H. 2016. Long non-coding RNA Unigene56159 promotes epithelial-mesenchymal transition by acting as a ceRNA of miR-140-5p in hepatocellular carcinoma cells. Cancer letters 382:166-175 DOI 10.1016/j.canlet.2016.08.029.

Ma X, Shen D, Li H, Zhang Y, Lv X, Huang Q, Gao Y, Li X, Gu L, Xiu S, Bao X, Duan J, Zhang X. 2015. MicroRNA-185 inhibits cell proliferation and induces cell apoptosis by targeting VEGFA directly in von Hippel-Lindau-inactivated clear cell renal cell carcinoma. Urologic oncology 33:169.e1-11 DOI 10.1016/j.urolonc.2015.01.003.

Martens-Uzunova ES, Böttcher R, Croce CM, Jenster G, Visakorpi T, Calin GA. 2014. Long noncoding RNA in prostate, bladder, and kidney cancer. European urology 65:1140-1151 DOI 10.1016/j.eururo.2013.12.003.

Qiao HP, Gao WS, Huo JX, Yang ZS. 2013. Long non-coding RNA GAS5 functions as a tumor suppressor in renal cell carcinoma. Asian Pacific journal of cancer prevention : APJCP 14:1077-1082.

Qin C, Han Z, Qian J, Bao M, Li P, Ju X, Zhang S, Zhang L, Li S, Cao Q, Lu Q, Li J, Shao P, Meng X, Zhang W, Yin C. 2014. Expression pattern of long non-coding RNAs in renal cell carcinoma revealed by microarray. PloS one 9:e99372 DOI 10.1371/journal.pone.0099372.

Qu L, Ding J, Chen C, Wu ZJ, Liu B, Gao Y, Chen W, Liu F, Sun W, Li XF, Wang X, Wang Y, Xu ZY, Gao L, Yang Q, Xu B, Li YM, Fang ZY, Xu ZP, Bao Y, Wu DS, Miao X, Sun HY, Sun YH, Wang HY, Wang LH. 2016. ExosomeTransmitted lncARSR Promotes Sunitinib Resistance in Renal Cancer by Acting as a Competing Endogenous RNA. Cancer cell 29:653-668 DOI 10.1016/j.ccell.2016.03.004.

Sakai I, Miyake H, Fujisawa M. 2013. Acquired resistance to sunitinib in human renal cell carcinoma cells is mediated by constitutive activation of signal transduction pathways associated with tumour cell proliferation. BJU international 112:E211-220 DOI 10.1111/j.1464-410X.2012.11655.x.

Salmena L, Poliseno L, Tay Y, Kats L, Pandolfi PP. 2011. A ceRNA hypothesis: the Rosetta Stone of a hidden RNA language. Cell 146:353-358 DOI 10.1016/j.cell.2011.07.014.

Sana J, Faltejskova P, Svoboda M, Slaby O. 2012. Novel classes of non-coding RNAs and cancer. Journal of translational medicine 10:103 DOI 10.1186/1479-5876-10-103.

Schmidt LS, Linehan WM. 2016. Genetic predisposition to kidney cancer. Seminars in oncology 43:566-574 DOI 
10.1053/j.seminoncol.2016.09.001.

Schmitt AM, Chang HY. 2016. Long Noncoding RNAs in Cancer Pathways. Cancer cell 29:452-463 DOI 10.1016/j.ccell.2016.03.010.

Serghiou S, Kyriakopoulou A, Ioannidis JP. 2016. Long noncoding RNAs as novel predictors of survival in human cancer: a systematic review and meta-analysis. Molecular cancer 15:50 DOI 10.1186/s12943-016-0535-1.

Siegel RL, Miller KD, Jemal A. 2017. Cancer Statistics, 2017. CA: a cancer journal for clinicians 67:7-30 DOI $10.3322 /$ caac. 21387 .

Su H, Sun T, Wang H, Shi G, Zhang H, Sun F, Ye D. 2017. Decreased TCL6 expression is associated with poor prognosis in patients with clear cell renal cell carcinoma. Oncotarget 8:5789-5799 DOI 10.18632/oncotarget.11011.

Vausort M, Wagner DR, Devaux Y. 2014. Long noncoding RNAs in patients with acute myocardial infarction. Circulation research 115:668-677 DOI 10.1161/CIRCRESAHA.115.303836.

Wang H, Niu L, Jiang S, Zhai J, Wang P, Kong F, Jin X. 2016. Comprehensive analysis of aberrantly expressed profiles of lncRNAs and miRNAs with associated ceRNA network in muscle-invasive bladder cancer. Oncotarget 7:86174-86185 DOI 10.18632/oncotarget.13363.

Wang L, Cai Y, Zhao X, Jia X, Zhang J, Liu J, Zhen H, Wang T, Tang X, Liu Y, Wang J. 2015. Down-regulated long noncoding RNA H19 inhibits carcinogenesis of renal cell carcinoma. Neoplasma 62:412-418 DOI 10.4149/neo_2015_049.

Wang Q, Zhang J, Liu Y, Zhang W, Zhou J, Duan R, Pu P, Kang C, Han L. 2016. A novel cell cycle-associated lncRNA, HOXA11-AS, is transcribed from the 5-prime end of the HOXA transcript and is a biomarker of progression in glioma. Cancer letters 373:251-259 DOI 10.1016/j.canlet.2016.01.039.

Wettersten HI, Aboud OA, Lara PN, Weiss RH. 2017. Metabolic reprogramming in clear cell renal cell carcinoma. Nature reviews. Nephrology 13:410-419 DOI 10.1038/nrneph.2017.59.

Xiao H, Tang K, Liu P, Chen K, Hu J, Zeng J, Xiao W, Yu G, Yao W, Zhou H, Li H, Pan Y, Li A, Ye Z, Wang J, Xu H, Huang Q. 2015. LncRNA MALAT1 functions as a competing endogenous RNA to regulate ZEB2 expression by sponging miR-200s in clear cell kidney carcinoma. Oncotarget 6:38005-38015 DOI 10.18632/oncotarget.5357.

Yang KC, Yamada KA, Patel AY, Topkara VK, George I, Cheema FH, Ewald GA, Mann DL, Nerbonne JM. 2014. Deep RNA sequencing reveals dynamic regulation of myocardial noncoding RNAs in failing human heart and remodeling with mechanical circulatory support. Circulation 129:1009-1021 DOI 10.1161/CIRCULATIONAHA.113.003863.

Yang S, Ning Q, Zhang G, Sun H, Wang Z, Li Y. 2016. Construction of differential mRNA-lncRNA crosstalk networks based on ceRNA hypothesis uncover key roles of lncRNAs implicated in esophageal squamous cell carcinoma. Oncotarget 7:85728-85740 DOI 10.18632/oncotarget.13828.

Yue B, Cai D, Liu C, Fang C, Yan D. 2016. Linc00152 Functions as a Competing Endogenous RNA to Confer Oxaliplatin Resistance and Holds Prognostic Values in Colon Cancer. Molecular therapy : the journal of the American Society of Gene Therapy 24:2064-2077 DOI 10.1038/mt.2016.180.

Zhang HM, Yang FQ, Chen SJ, Che J, Zheng JH. 2015. Upregulation of long non-coding RNA MALAT1 correlates with tumor progression and poor prognosis in clear cell renal cell carcinoma. Tumour biology : the journal of the International Society for Oncodevelopmental Biology and Medicine 36:2947-2955 DOI 10.1007/s13277-014-2925-6.

Zubakov D, Stupar Z, Kovacs G. 2006. Differential expression of a new isoform of DLG2 in renal oncocytoma. BMC cancer 6:106 DOI 10.1186/1471-2407-6-106. 
463 Supporting information

464 Table 1.RNAs significantly associated with overall survival in the lncRNA-miRNA-mRNA pathway.

465

466 Figure legends

467 Figure1. Flow chart of the ceRNA network construction.

468 Figure2.Venn diagram of aberrant expression profiles of $\operatorname{lncRNAs}(\mathrm{A})$, miRNAs(B) and mRNAs(C) between stage I RCC group (tumor tissues according to AJCC)/control group (non-tumor tissues), stage II group/control group, stage III group/control group, and stage IV group/control group. A total of 291 intersecting lncRNAs, 102 intersecting miRNAs and 2269 mRNAs were identified.

472 Figure 3. Gene ontology (GO) and Kyoto Encyclopedia of Genes and Genomes (KEGG) pathway analyses. A. 473 Top 10 processes of up- and down-regulated genes in the GO analysis. B. Top 10 pathways of up- and down474 regulated genes in the pathway analysis.

475 Figure 4.The miRNA-IncRNA-mRNA ceRNA network is composed of 106 lncRNAs, 26 miRNAs and 69 mRNAs.Red diamonds represent up-regulated miRNAs, while green diamonds represent down-regulated miRNAs. Triangles and circles represent lncRNAs and mRNAs, respectively, while red indicates up-regulation, and green indicates down-regulation. Figure5. The protein-protein interaction networkincluded 44 genes with scores of $>0.4$ in String. Figure6. Kaplan-Meier survival curves for 11 related RNAs (4 lncRNAs, 3 miRNAs and 4 mRNAs) in the ceRNA network (horizontal axis: overall survival times: days, vertical axis: survival function).

\section{Supplementary material}

Supplementary Figure 1. Heat map depicting the differential expression profiles of the intersecting lncRNAs. The horizontal axis on top shows the sample names. The right vertical axis displays the names of the lncRNAs, while the left vertical axis represents gene clustering. The expression values are described by a color scale, in which red indicates high expression, while green indicates low expression.

Supplementary Table 1. Top ten up-regulated and down-regulated intersection lncRNAs.

Supplementary Table 2. Top 20 GO gene sets correlate with up-regulated mRNAs by GSEA 
495

496

497

498 


\section{Figure 1}

\section{Flow chart of the ceRNA network construction.}

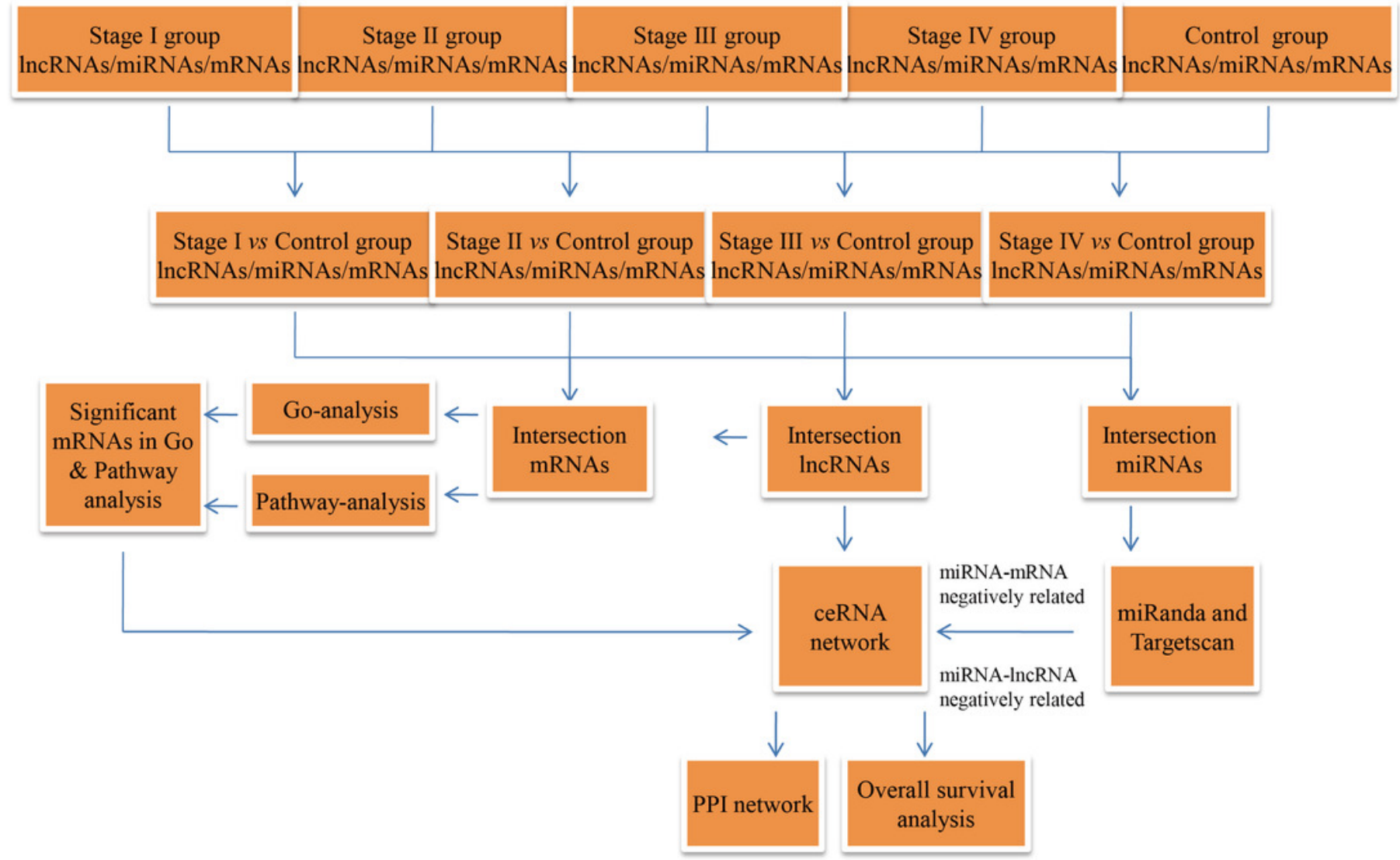


Figure 2

\section{Venn diagram of aberrant expression profiles}

Venn diagram of aberrant expression profiles of IncRNAs(A), miRNAs(B) and mRNAs(C) between stage I RCC

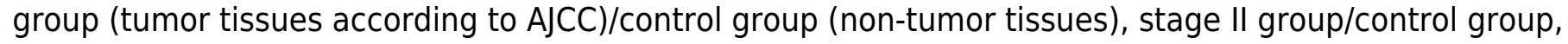
stage III group/control group, and stage IV group/control group. A total of 291 intersecting IncRNAs, 102 intersecting miRNAs and 2269 mRNAs were identified.

A

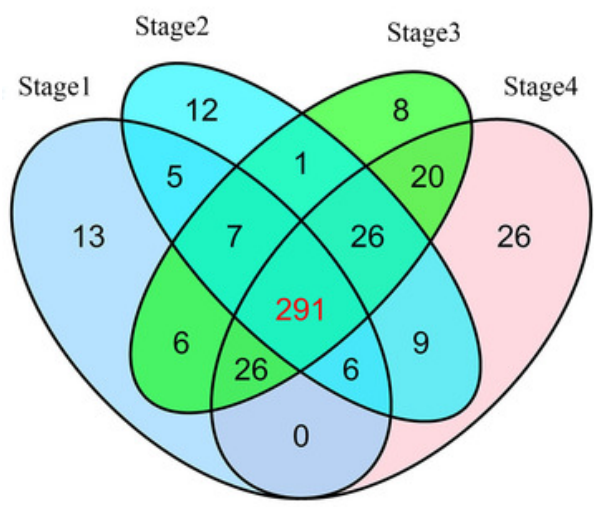

lncRNA
B

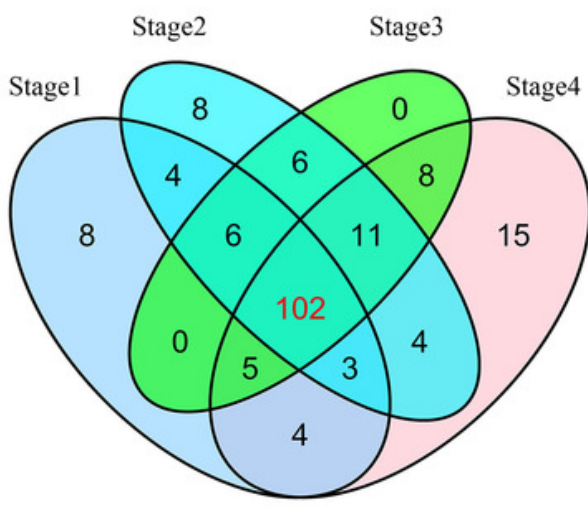

miRNA
C

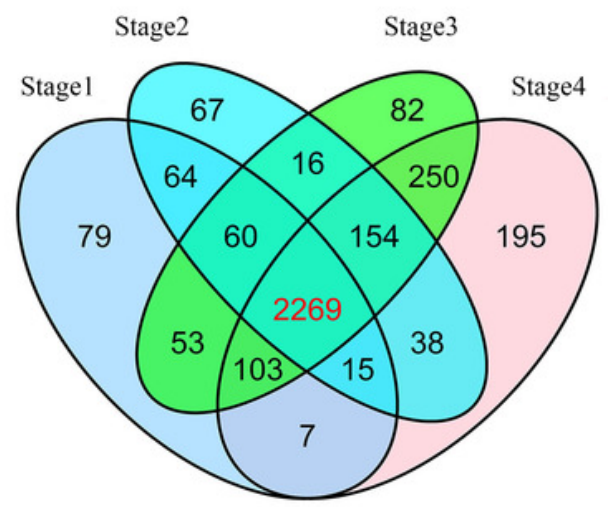

mRNA 
Figure 3

\section{Gene ontology (GO) and Kyoto Encyclopedia of Genes and Genomes (KEGG) pathway analyses.}

\section{A. Top 10 processes of up- and down-regulated genes in the GO analysis. B. Top 10 pathways} of up- and down-regulated genes in the pathway analysis.

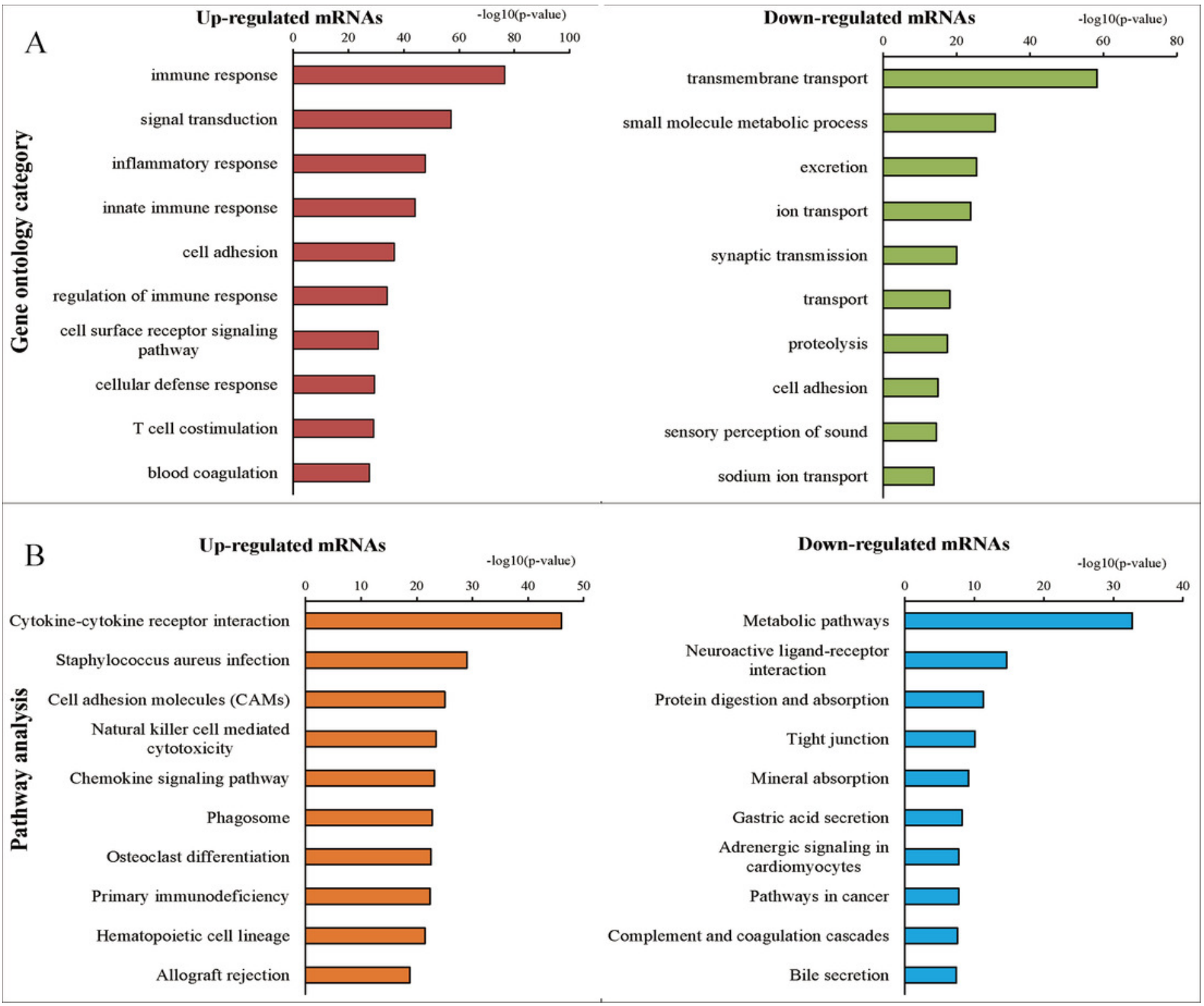




\section{Figure 4}

The miRNA-IncRNA-mRNA ceRNA network is composed of 106 IncRNAs, 26 miRNAs and 69 mRNAs.

Red diamonds represent up-regulated miRNAs, while green diamonds represent downregulated miRNAs. Triangles and circles represent IncRNAs and mRNAs, respectively, while red indicates up-regulation, and green indicates down-regulation.

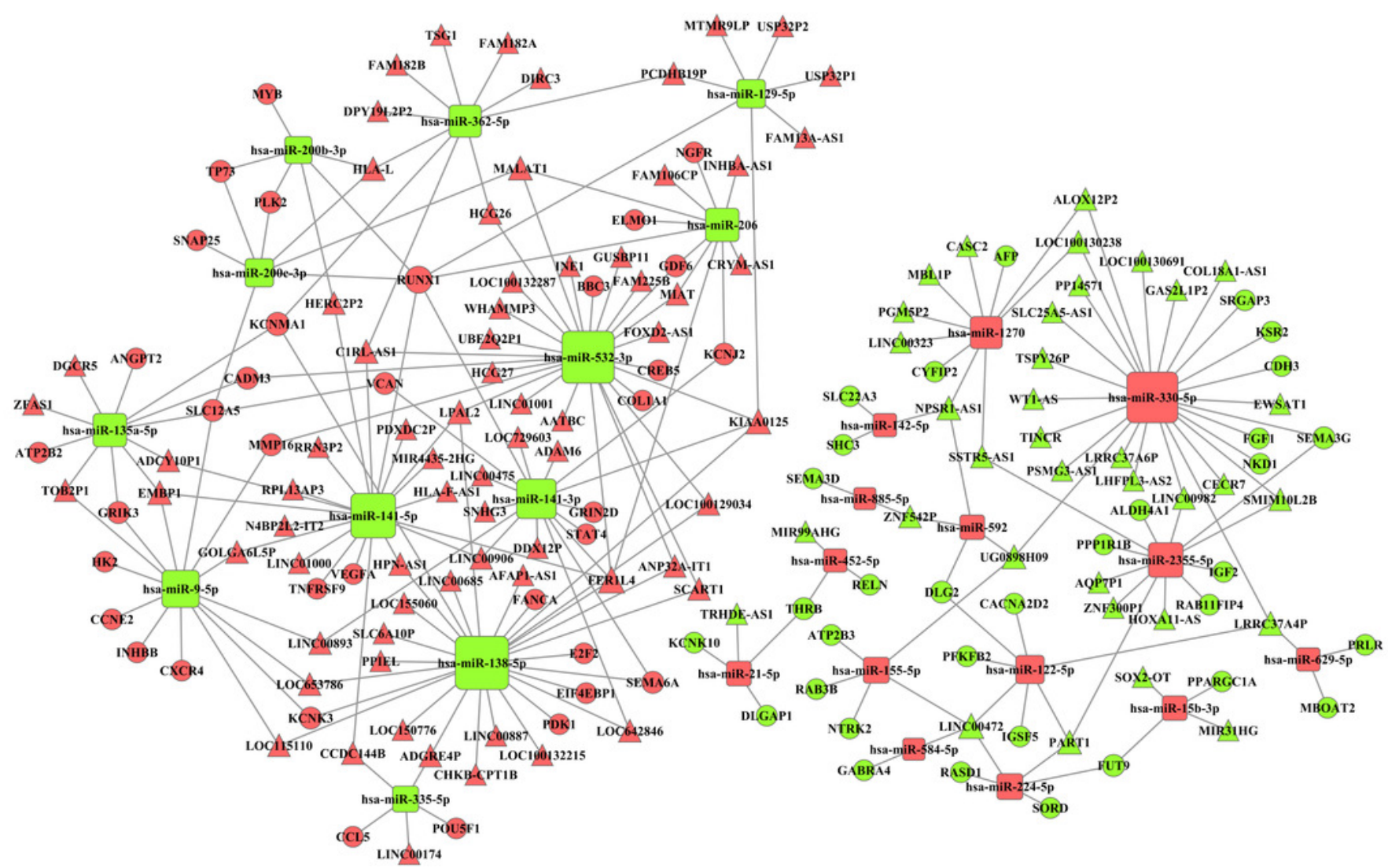


Figure 5

The protein-protein interaction network included 44 genes with scores of $>0.4$ in String.

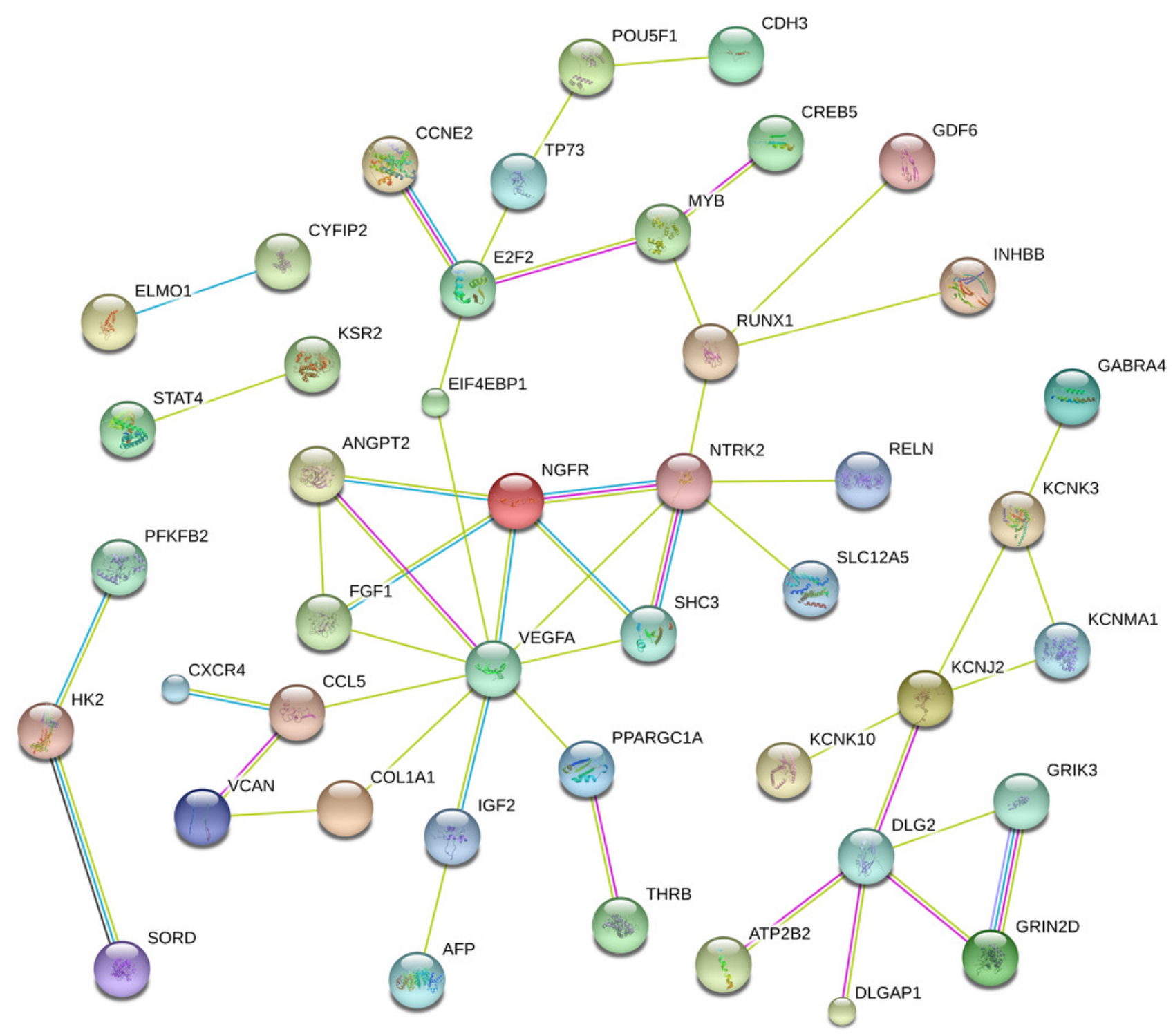




\section{Figure 6}

Kaplan-Meier survival curves for 11 related RNAs ( 4 IncRNAs, 3 miRNAs and 4 mRNAs) in the ceRNA network (horizontal axis: overall survival times: days, vertical axis: survival function).
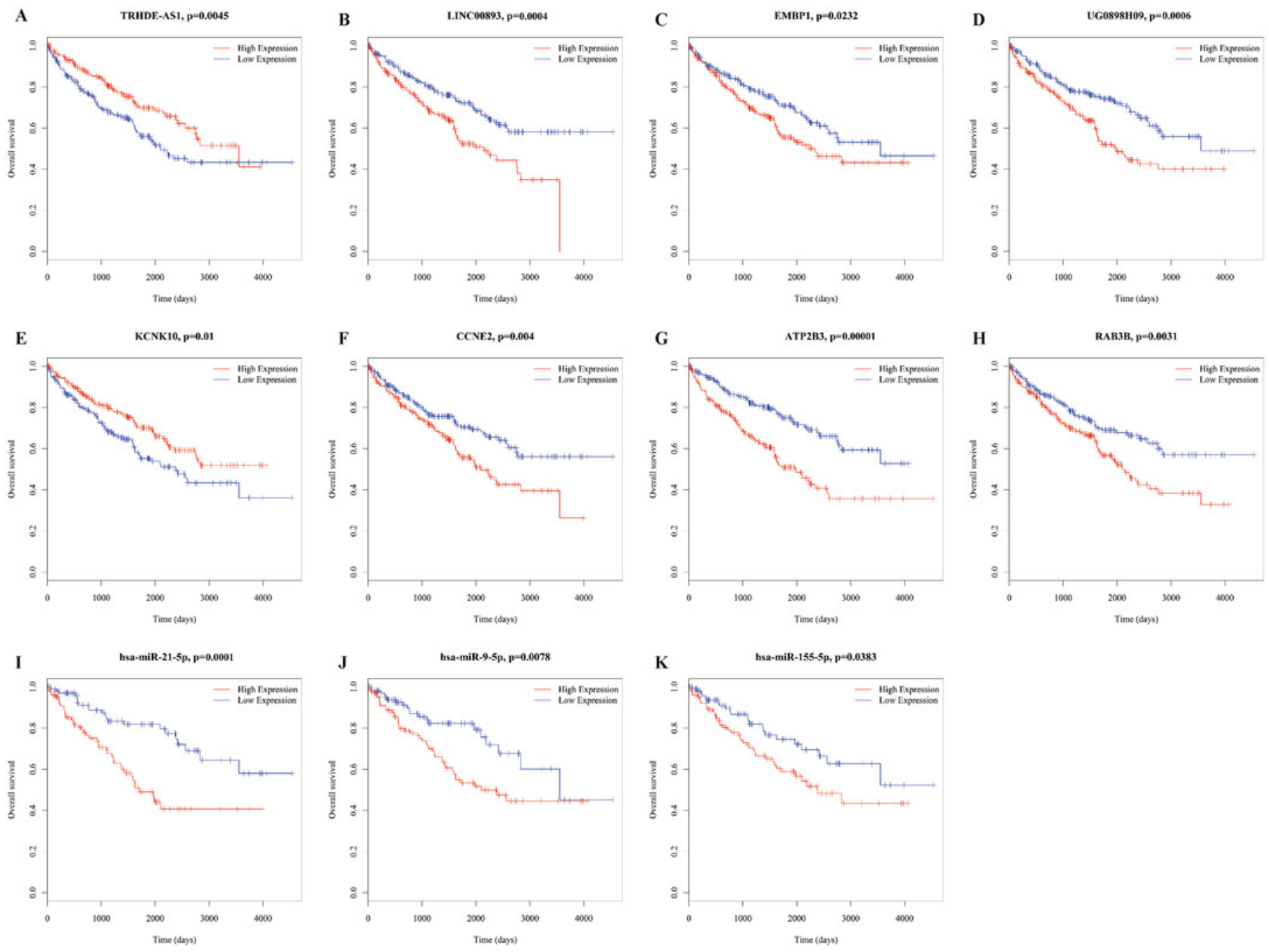


\section{Table $\mathbf{1}$ (on next page)}

RNAs significantly associated with overall survival in the IncRNA-miRNA-mRNA pathway. 
1 Table 1. RNAs significantly associated with overall survival in lncRNA-miRNA-mRNA pathway.

\begin{tabular}{lll}
\hline IncRNA & miRNA & mRNA \\
\hline TRHDE-AS & miR-21-5p & KCNK10 \\
Lin00893 & miR-9-5p & CCNE2 \\
EMBP1 & miR-9-5p & CCNE2 \\
UG0898H09 & miR-155-5p & ATP2B3 \\
UG0898H09 & miR-155-5p & PAB3B \\
\hline
\end{tabular}

2

3 